\title{
Spatial Relations Analysis by Using Fuzzy Operators
}

\author{
Nadeem Salamat and El-hadi Zahzah \\ Université de La Rochelle \\ Laboratoire de Mathématiques, Images et Applications \\ Avenue M Crépeau La Rochelle 17042, France \\ \{nsalam01, ezahzah\}@univ-lr.fr \\ nadeemsalamat@hotmail.com
}

\begin{abstract}
Spatial relations play important role in computer vision, scene analysis, geographic information systems (GIS) and content based image retrieval. Analyzing spatial relations by Force histogram was introduced by Miyajima et al [1] and largely developed by Matsakis [2] who used a quantitative representation of relative position between $2 \mathrm{D}$ objects. Fuzzy Allen relations are used to define the fuzzy topological relations between different objects and to detect object positions in images. Concept for combined extraction of topological and directional relations by using histogram was developed by J.Malki and E.Zahzah [3], and further improved by Matsakis [4]. This algorithm has high computational and temporal complexity due to its limitations of object approximations. In this paper fuzzy aggregation operators are used for information integration along with polygonal approximation of objects. This approach gives anew, with low temporal and computational complexity of algorithm for the extraction of topological and directional relations.
\end{abstract}

Keywords: Spatial Relations, Force Histogram, Polygonal Approximation, Temporal Complexity, Fuzzy aggregation operators.

\section{Introduction}

Space relations has a remarkable importance in computer vision and image analysis as in content based image retrieval, similarity based image retrieval, identify forms, manage data bases, support spatial data in artificial intelligence (AI), cognitive science, perceptual psychology, geography particularly geo-information system (GIS), indexation and comparing objects scene and model are major applications of space relations. Different approaches for finding spatial and topological relations have been developed according to the need for applications and object representations. Qualitative methods for directional and topological relations includes Max J. Egenhofer's method of four and 9 intersections [56]. These methods are considered most important in GIS community. Directional relations are defined on relative frame of reference and absolute frame of reference. In relative frame of reference position of a simple object is made with respect to an 
oriented line or an ordered set forming a vector to some intrinsic properties of reference object. Methods like angle histogram introduced by K.Miyajima and A.Ralescu [1] and statistical method developed by MinDeng.Zalimli [7, $R$ - histogram [8] depends upon relative reference frame. Matsakis [2] introduced $1 \mathrm{D}$ representation of $2 \mathrm{~d}$ objects by the union of longitudinal sections which is the extension of angle histogram. The derivation of cobined topological and directional relations by using force histogram [4] was first introduced by J.Malki and E.Zahzah [3], then Matsakis raised some problems regarding fuzziness of some relations like meet and meet_by and some others which exist at segmentation level. In case of longitudinal section fuzzification process introduced by Matsakis 4 restricts the object approximation which increases the temporal and computational complexity of algorithm.

Approximating the object by its boundary, length of longitudinal sections can be computed as distance between the intersecting points of oriented line and object boundary. The degree of fuzzy membership function depends upon three values $x, y$ and $z$. In tuple $(x, y, z)$, the pair $(x, z)$ are the lengths of longitudinal sections and $y$ is the difference between maximum value of intersecting points of object $B$ and minimum value of object $A$, i.e. $y \in R$ or $y \in Z$. By this approach of object approximation, temporal complexity decreases from $n \sqrt{n}$ to $N \log (N)$ where $n$ is number of pixels of objects under consideration and $N$ is the number of vertex of object polygons. Temporal complexity for the said algorithm is not given but in general temporal complexity of force histograms is discussed in 9] for different object types. We assume same temporal complexity for objects because segmentation level problems raised by Matsakis forced the object as raster data and in addition to this algorithm for fuzzification of longitudinal sections increases temporal and computational complexity. These problems no more exist if we consider objects by their boundary, then need for Matsakis's algorithm remain for objects having disconnected boundaries. Each segment is separated by a certain distance. This internal distance has a significant impact on directional and topological relations. Fuzzy disjunctive operators are used. These fuzzy operators have been developed to summarize information distributed in different sets by grades of fuzzy membership values. This paper is structured as follows. First of all we describe Allen relations in space. In section 2 we describe different fuzzy

Allen relations defined by Matsakis and changes in mathematical formulation of fuzzy histogram of Allen relations due to object approximation. In section 3 we discuss different fuzzy operators, section 4 describes experimental results. In section 5 temporal complexity is calculated and section 6 concludes the paper.

\section{Allen Relations in Space}

Allen [10, introduced the well known 13 mutually exclusive exhaustive interval relations based on temporal interval algebra. These relations are arranged as $A=$ $\left\{<, m, o, s, f, d, e q, d_{i}, f_{i}, s_{i}, o_{i}, m_{i},>\right\}$. where $\{<, m, o, s, f, d\} \quad,\left(\left\{d_{i}, f_{i}, s_{i}, o_{i}\right.\right.$, $\left.m_{i},>\right\}$ ) are the relation before, meet, overlap, start, finish, during (resp the inverse relations of the cited ones). The relation $e q$ is the equality spatial relation. 


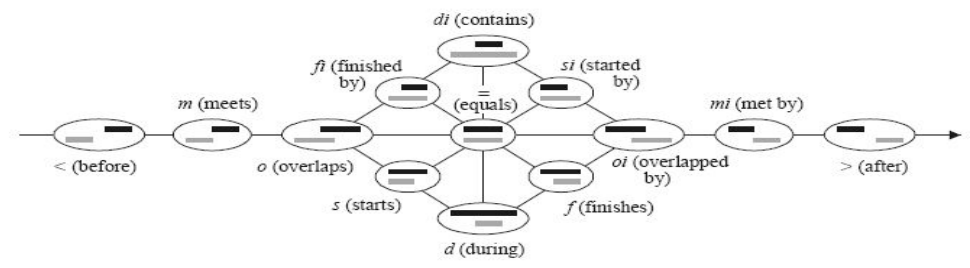

Fig. 1. Black segment represents the reference object and gray segment represents argument object. figure extracted from [2].

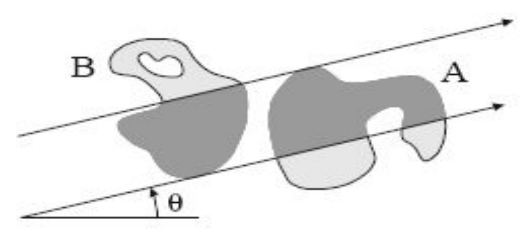

Fig. 2. Histogram of Allen in direction $\theta$ ( dark gray area represents histogram of fuzzy Allen relations, figure extracted from [2])

All the Allen relations in space are conceptually illustrated in figure 2 These relations have a rich support for the topological and directional relations.

\section{Fuzzy Histogram of Allen Relations}

In real applications, small errors in crisp values can change the entire result when gradual changes of topological relations occur over time. To cope these problems fuzzification was introduced, it comprises the process of transforming crisp values into grades of membership for linguistic terms of fuzzy sets. Fuzzification process of Allen relations do not depend upon particular choice of fuzzy membership function, trapezoidal membership function is used due to flexibility in shape change. Let $r(I, J)$ is Allen relation between segments $I$ and $J$ where $I \in A$ (argument object) and $J \in B$ (reference object), $r^{\prime}$ is the distance between $r(I, J)$ and its conceptional neighborhood. We consider a fuzzy membership function $\mu: r^{\prime} \longrightarrow[0,1]$. The different fuzzy Allen relations defined by Matsakis [4] are

$$
\begin{gathered}
f_{b}(I, J)=\mu_{(-\infty,-\infty,-b-3 a / 2,-b-a)}(y) \\
f_{m}(I, J)=\mu_{(-b-3 a / 2,-b-a,-b-a,-b-a / 2)}(y) \\
f_{f}(I, J)=\min \left(\mu_{(-(b+a) / 2,-a,-a,+\infty)}(y), \mu_{(-3 a / 2,-a,-a,-a / 2)}(y), \mu_{(-\infty,-\infty, z / 2, z)}(x)\right) \\
f_{f i}(I, J)=\min \left(\mu_{-b-a / 2,-b,-b,-b+a / 2}(y), \mu_{(-\infty,-\infty,-(b+a) / 2)}(y), \mu_{(z, 2 z,+\infty,+\infty)}(x)\right) \\
f_{d}(I, J)=\min \left(\mu_{(-b,-b+a / 2,-3 a / 2,-a)}(y), \mu_{(-\infty,-\infty, z / 2, z)}(x)\right) \\
f_{d i}(I, J)=\min \left(\mu_{(-b,-b+a / 2,-3 a / 2,-a)}(y), \mu_{(z, 2 z,+\infty,+\infty)}(x)\right)
\end{gathered}
$$


where $a=\min (x, z), b=\max (x, z), x$ is the length of longitudinal section of argument object $A$, and $z$ is the length of longitudinal section of reference object $B$. Most of relations are defined by one membership function and some of them by the minimum value of more than one membership functions like $d$ (during), $d_{i}\left(\right.$ during_by), $f$ (finish), $f_{i}$ (finished_by). In fuzzy set theory, sum of all the relations is one, this gives the definition for fuzzy relation equal. Histogram of fuzzy Allen relations stated by Matsakis 44 is "Histogram of fuzzy Allen relations represents the total area of subregions of $A$ and $B$ that are facing each other in given direction $\theta^{\prime \prime}$.

In this new approach, fuzzy Allen relations are computed for each segment. Fuzzy Allen relation for each segment is a fuzzy set and fuzzy aggregation operators are used to combine different values of fuzzy grades. This results the change in above definition of fuzzy histogram of Allen relations. Mathematically this becomes

$$
\int_{-\infty}^{+\infty}\left(\sum_{r \in A} F_{r}\left(q, A_{q}(v), B_{q}(v)\right) d v=(x+z) \sum_{k=1}^{n} r\left(I_{k}, J_{k}\right)\right.
$$

where $z$ is the area of reference object and $x$ is area of augmented object in direction $\theta, n$ is total number of segments treated and $r\left(I_{k}, J_{k}\right)$ is an Allen relation for segments $I_{k}, J_{k}$.

\section{Fuzzy Operators and Treatment of Longitudinal Sections}

During the decomposition process of an object into segments, there can be multiple segments depending on object shape and boundary which is called longitudinal section. Different segments of a longitudinal section are at a certain distance and these distances might effect end results. After polygon object approximation, we need for the fuzzification algorithm when object has disconnected boundary. In this case there exist number of $1 \mathrm{D}$ segments of concave object or object having disconnected boundary. Each segment and its distance from other segment has its own impact on fuzzy Allen relations of whole object. To cope with this, fuzzy operators are used. In literature of fuzzy set theory there exist variety of operators such as fuzzy $T$-norms, $T$-conorms and so on, which can be used for fuzzy integration of available information. Some mostly used operators for data integration in [1] are:

$\mu_{(O R)}(u)=\max \left(\mu_{(A)}(u), \mu_{(B)}(u)\right) ; \mu_{(A N D)}(u)=\min \left(\mu_{(A)}(u), \mu_{(B)}(u)\right) ;$ $\mu_{(S U M)}(u)=1-\Pi_{i=1}^{2}\left(\mu_{(i)}(u)\right)$,

When fuzzy operator $O R$ (respectively $A N D$ ) is used, only one fuzzy value contributes for the resultant value which is maximum (respectively minimum). For other operators both values contribute. In this case each Allen relation has a fuzzy grade objective is to accumulate the best available information. In case of longitudinal section, there exist number of segments and each segment has 
a fuzzy Allen relation with segment of other object. Suppose that longitudinal section of object $B$ has two segments such that $z=z_{1}+z_{2}$ where $z_{1}$ is the length of first segment and $z_{2}$ is the length of second segment and $z$ is length of longitudinal section. Let $\mu_{1}\left(y_{1}\right)$ defines the value of fuzzy Allen relations with the first segment and $\mu_{2}\left(y_{2}\right)$ represents value of fuzzy Allen relations with the second segment where $y_{1}$ and $y_{2}$ are the distances between object $A$ and two segments of $B$. Now Fuzzy $O R$ operator is used to get consequent information obtained from two sets of fuzzy Allen relations.

\section{$5 \quad$ Experiments and Interpretation}

For the experiment purpose 360 directions are considered (angle increment is 1 degree) and lines are drawn by $2 \mathrm{~d}$ Bresenham digital line algorithm. Instead of considering all the $v$ values, only those lines are considered which passes through vertex of polygon. segments are computed and fuzzy Allen relations are computed for each segment, if there exit longitudinal section then fuzzy aggregation operator is applied to obtain the resultant fuzzy Allen relation of whole object. Each relation is associated with the gray scale value like before with black and white represents after. Same notations as Matsakis are used except changing the boundary color of a relation for better visualization of relations. Opposite relations have the same boundary color such as $m$ (meet) and (meet_by) relations have the yellow boundary. Object $A$ has the light gray color while object $B$ is represented by dark gray. The thirteen histograms that represent directional and topological relations are represented in the layers and each vertical layer represents total area of objects in that direction. Here histograms are not normalized. All relations are symmetric in nature except $d($ during $)$ and di(during_by).

$f_{b}^{A B}(\theta)=f_{a}^{A B}(\theta+\pi), f_{m i}^{A B}(\theta)=f_{m}^{A B}(\theta+\pi), f_{o i}^{A B}(\theta)=f_{o}^{A B}(\theta+\pi)$, $f_{s i}^{A B}(\theta)=f_{f i}^{A B}(\theta+\pi), f_{f}^{A B}(\theta)=f_{s}^{A B}(\theta+\pi)$ and for $d($ during $)$, di(during_by) it will be $f_{d}^{A B}(\theta)=f_{d i}^{B A}(\theta)$.

In fig 3(a) explains the representation of fuzzy Allen histograms.In fig 3(b) Shows the representation of histograms and explains that each relation is represented by a layer and each layer have a different gray level color associated with a relation, boundary color is not represented here. Same colors association with a relation is used only boundary colors are changed. In fig 3(c) represents object position where $A$ is light gray object and object $B$ is represented by dark gray color. Fig 3(c) represents histogram associated with objects pair, where y axis represents total area of objects having different relations and directions are represented along $\mathrm{x}$ axis. At a certain value $f$ represents area under the finish relation and $d$ represents area having during relation and total area is sum of both areas. Different set of examples are considered, in first case both the objects are convex and second case argument object $A$ is convex and reference 


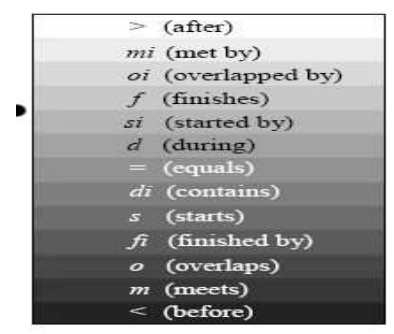

(a)

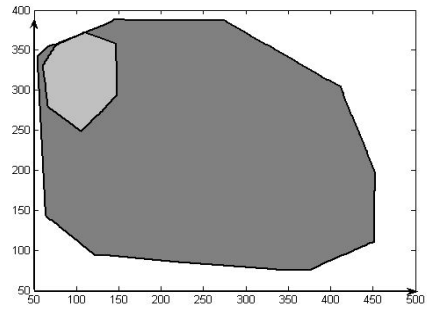

(b)

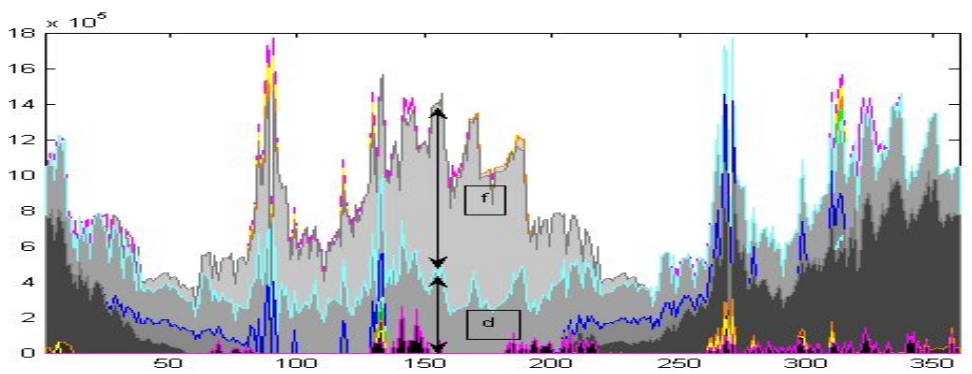

(c)

Fig. 3. (a)Explanation of gray level value associated with a relation( source: 44) (b)Object pair representation. (c) Corresponding histogram.

object $B$ is concave. In this experiment Fig 4(a) Pair of objects under consideration are at enough distance. Fig 4(b) represents the corresponding fuzzy histogram of Allen relations, at this stage only after and before fuzzy relations exist. Fig 4(e) object $A$ moves to words center of object $B$ and it overlaps $B$. Fig $4(\mathrm{~d})$ represents its histogram at this almost all the relations exist. Fig 4(e) position of object $A$ is at center of object $B$. Fig $4(\mathrm{f})$ represents its histogram during relation exist, There exist after and be fore relation near the diagonal direction. Which is due to zigzag of lines in digital space. In this set of examples objects are taken at different distances to show that the relations are sensitive to distance between them and their sensitivity also depends upon relative size.

Now for second set of examples. In this example rectangular objects are considered. Object $A$ firstly for away from the $U$ shaped object $B$. Fuzzy Allen relations are calculated separately for each segment then fuzzy operator is used. Main objective of this example is to show that each segment of longitudinal section has its own impact on fuzzy Allen relation and each segment may have same, opposite neither opposite nor same Allen relations as in case of fig $5(\mathrm{a})$ to fig 5(c). In Fig 5(a) object $A$ is at a certain distance to object $B$. Fig $5(\mathrm{~b})$ only after and before relation exists because both parts of object $B$ has the same relation. Finally in fig $5(\mathrm{c})$ when object $A$ is between two parts of object $B$, 


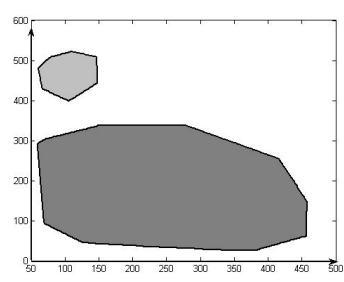

(a)

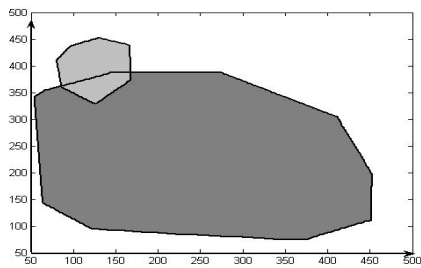

(c)

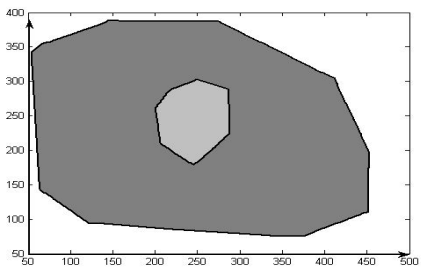

(e)

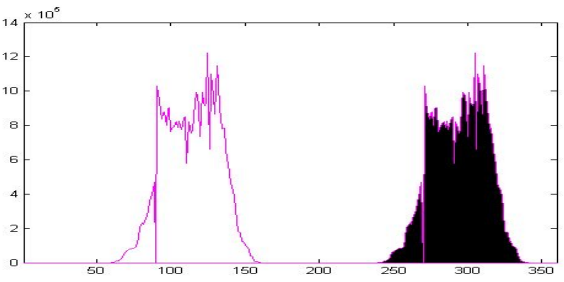

(b)

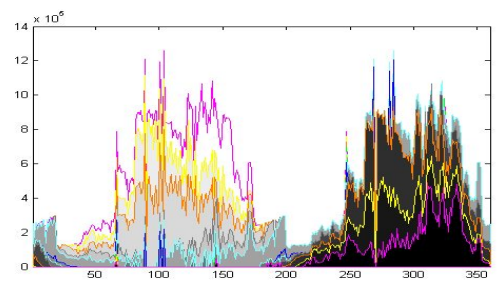

(d)

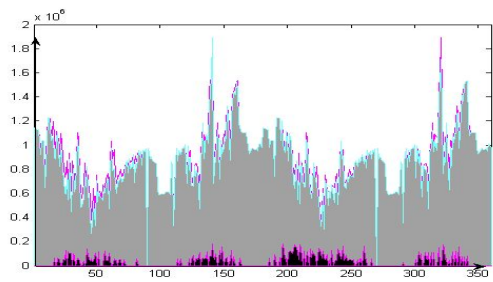

(f)

Fig. 4. Pairs of convex objects (where along x-axis angle is zero degree ) and their corresponding fuzzy histogram of Allen relations

both segments have opposite relations before and after meanwhile there exist relation during which is due to zigzag phenomena of digital space and line algorithm.

\section{Temporal Complexity}

Finding the exact temporal complexity is a tough job, major aim of this study is to find time length required by the algorithm. To expresse the computational time as a function of $N$ a language is required which grows on the order of $N$. Five symbols for comparing rates are used such as $o, O, \theta, \Omega$ and $\sim$. In fact asymptomatically equality is an formalism of idea to find the conditions that two functions have same growth rate.i.e. limit $_{n \rightarrow \infty}\left(\frac{a_{n}}{b_{n}}\right)=1$ and $a_{n}=O\left(b_{n}\right)$ if $\left|\frac{a_{n}}{b_{n}}\right|$ is bounded. Asymptotic analysis algorithm is used. For this purpose a function which represents upper bound of function used to represent the algorithm complexity is found. In this case time constraint depends upon length of line, 


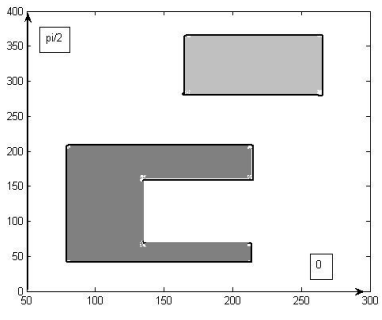

(a)

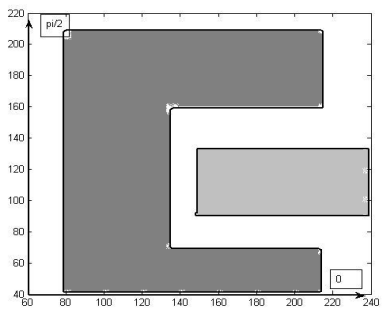

(c)

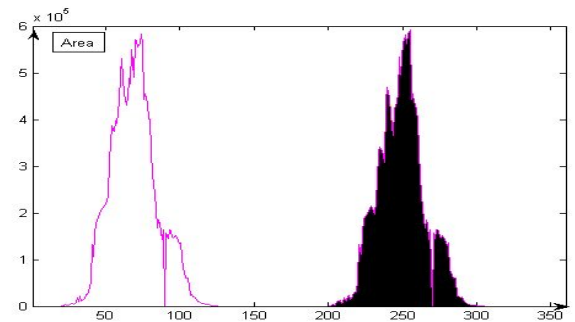

(b)

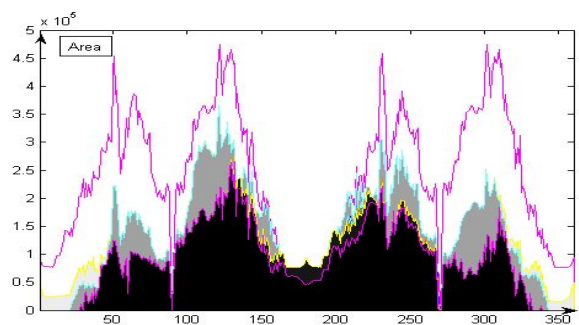

(d)

Fig. 5. Convex and concave pair of objects (where along x-axis angle is zero degree ) and their corresponding fuzzy histogram of Allen relations

contour length and number of polygon vertexes. Time (round in seconds) for all 360 directions is used when line length is fixed to 1000, 1200, 1400 pixels. Following tables represent different computations where $\mathrm{L}$ is length of line and $\mathrm{N}$ represents number of polygon vertexes. There is a symmetry between the different values of cost function (Time function) in table 1 and table 2 and the number of polygons vertexes. By observing the graphical representation of data, (graph fig 6(a) ) in table 1 and graph fig 6(b) of data in table 2) each time graph for a fixed length of line and given objects sizes (length of contours). It seems that graph is displaced by a constant value of $T$ and its growth rate is less than $n \log (n)$ hence function $f(n)=n \log (n))$ representation the upper bound of our graphs. (Graphes given in figure (6) So histogram of fuzzy Allen relations are of order $O(N \log (N))$

Table 1. Contour of 1300 pixels

\begin{tabular}{|c|c|c|c|}
\hline$N \backslash L$ & 1000 & 1200 & 1400 \\
\hline 24 & 63 & 67.14 & 74 \\
\hline 25 & 66.5 & 73 & 78 \\
\hline 26 & 68 & 74.3 & 80 \\
\hline 27 & 72 & 79 & 84.34 \\
\hline 28 & 73.20 & 82 & 86.20 \\
\hline
\end{tabular}

Table 2. Contour 3300 of pixels

\begin{tabular}{|c|c|c|c|}
\hline$N \backslash L$ & 1000 & 1200 & 1400 \\
\hline 25 & 97.5 & 103 & 113.5 \\
\hline 26 & 102 & 107 & 115.5 \\
\hline 27 & 109 & 115.5 & 123 \\
\hline 28 & 113 & 119 & 126 \\
\hline 29 & 120 & 125 & 129.4 \\
\hline 30 & 124 & 129 & 135 \\
\hline
\end{tabular}




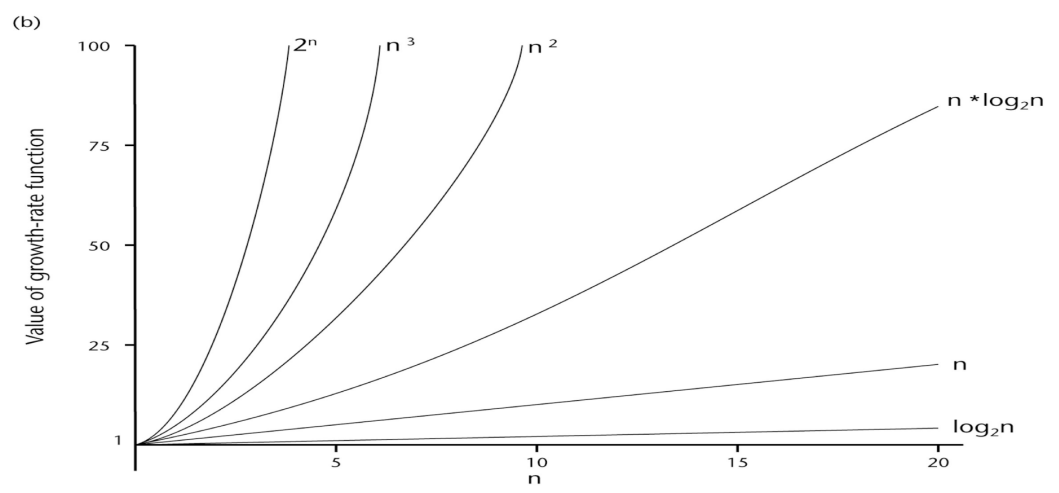

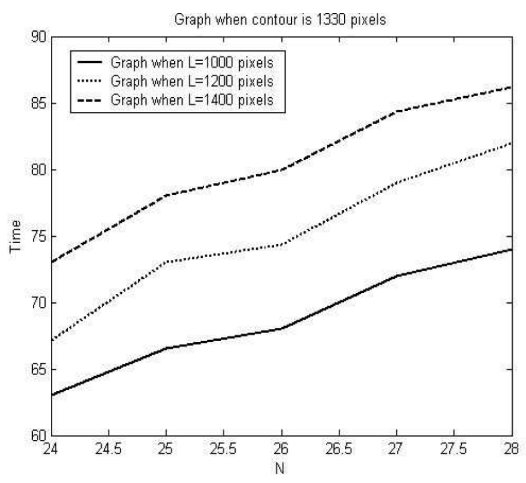

(a)

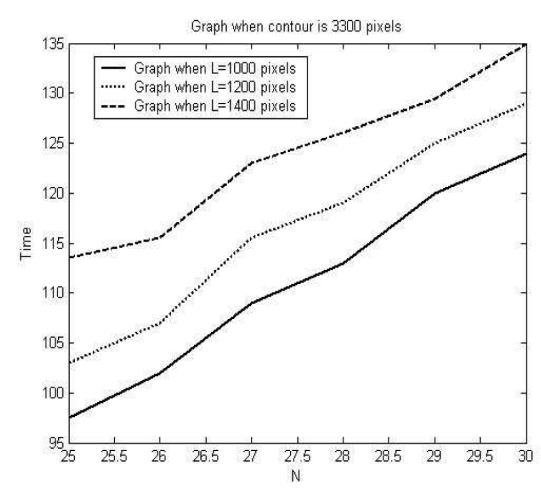

(b)

Fig. 6. (a)Graph of some known functions, (b)Graphs of data given in table No.1(c)Graphs of data given in table No.2 where time is rounded off in seconds

\section{Conclusion}

It is shown that histogram of fuzzy Allen relations associated with pair of objects carry a lot of information. To deal with concave objects or objects having disconnected boundaries, fuzzy operators are used. Use of these operators is simple so polygonal approximation of objects and application of fuzzy aggregation operator simplifies the algorithm given by Matsakis [4. This approach decrease its temporal and computational complexity due to avoiding the fuzzification process developed by Matsakis. Certainly this approach of using fuzzy operator will open new fields of applications for fuzzy aggregation operators. Here we calculated all the directions for experimental purpose, in practice we performed only limited number of directions according to the requirement of application. Allen relations are used for describing the relative object position in image understanding. 


\section{References}

1. Miyajima, K., Ralescu, A.: Spatial Organization in 2D Images, Fuzzy Systems. In: IEEE World Congress on Computational Intelligence, vol. 1, pp. 100-105 (1994)

2. Matsakis, P., Laurent Wendling, J.D.: Représentation de La Position Relative d' Objets $2 \mathrm{D}$ au Moyen $D^{\prime}$ Un Histogramme de Forces. Traitement du Signal 15, 25-38 (1998)

3. Malki, J., Zahzah, E., Nikitenko, D.: Indexation et Recherche d' Image Fondées Sur Les Relations Spatiales Entre Objets. Traitement du Signal 19(4), 235-250 (2002)

4. Matsakis, P.: Combined Extraction of Directional and Topological Relationship Information from 2D Concave Objects, in fuzzy modeling with spatial informations for geographic problems, New York, pp. 15-40 (2005)

5. Egenhofer, M.J., Franzosa, R.D.: Point Set Topological Relations. International Journal of Geographical Information Systems 5(2), 161-174 (1991)

6. Egenhofer, M.J., Sharma, J., Mark, D.M.: A Critical Comparison of The 4Intersection and 9-Intersection Models for Spatial Relations: Formal Analysis. Auto-Carto 11, 1-12 (1993)

7. Li, M.D.: A Statistical Model for Directional Relations Between Spatial Objects. GeoInformatica 12(2), 193-217 (2008)

8. Wang, Y., Makedon, F.: R-histogram:quantitative representation of spatial relations for similarity-based image retrieval. In: MULTIMEDIA 2003, pp. 323-326. ACM, New York (2003)

9. Pascal Matsakis, D.N.: Applying Soft Computing in Defining Spatial Relations, Understanding the Spatial Organization of Image Regions by Means of Force Histograms A Guided Tour, pp. 99-122. Springer, New York (2002)

10. Allen, J.F.: Maintaining Knowledge about Temporal Intervals. Communications of the ACM 26(11), 832-843 (1983)

11. Chi, K.-H., No-Wook Park, C.J.C.: Fuzzy Logic Intergration for Landslide Hazard Mapping using Spatial Data from Boeun, Korea. In: Symposium on geospatial theory, processing and application, ottawa 